\title{
Bariatric Surgery and Quality of Life
}

\author{
Ayiomamitis G
}

Quality of life (QOL) is defined as the impact of health on an individual's functioning, encompassing physical, psychological and social wellbeing; it is highly subjective, relying on personal experiences, beliefs and expectations [1]. Research consistently shows that obese individuals experience poorer physical and mental QOL than nonobese individuals [2].

Weight-related impairment of QOL is thought to be the combined result of the physical (e.g., pain, physical activity), psychological (e.g., self-esteem, self-motivation, depressive symptoms, disordered eating) and social (e.g., social support, weight-related stigmatization) impact of excess weight. Desire for improvement in QOL is often a motivator for seeking bariatric surgery and QOL is closely related to patient satisfaction following such surgery [3].

While there is evidence demonstrating a relationship between improvement in QOL and weight loss after bariatric surgery [4], this improvement cannot be explained by weight loss alone. Patients experience a marked improvement in perceived QOL immediately following surgery, even before any significant weight loss can occur, suggesting that some other factors, such as psychological and social elements, or even changes in the gut microbiota may interact with weight loss to influence the improvement in QOL following bariatric surgery [5].

The gut microbiota has captured our attention in the last decade as an element that directly affects our health or disease status, and in particular, it has been implicated in the etiology of obesity [6]. Gut microbiota is considered as an assortment of microorganisms that inhabit the gastrointestinal (GI) tract. The composition of this microbial community depends on the host, but it can also be modified by exogenous and endogenous events [7]. With regard to the host, these bacteria are symbiotic, and play an important role in physiological processes, for example, in digestion, or they can intervene in the metabolism, as they can increase energy production from the diet and take part in the regulation of the composition of fatty acid tissue [8]. Various different bacteria can also induce low-grade inflammation. All these processes are involved in the pathophysiology of obesity and metabolic disorders.

\footnotetext{
$1^{\text {st }}$ Department of Surgery, Tzaneio General Hospital, Piraeus, Greece

Corresponding author: Ayiomamitis D Georgios MD, MSc (MIS), PhD, FACS Consultant General and Laparoscopic Surgeon,

$1^{\text {st }}$ Dept of Surgery, Tzaneio General Hospital, Zanni and 1 Afentouli St., 18536, Piraeus, Greece

e-mails: agiogeo@gmail.com, info@lapexperts.com.cy
}

Laparoscopic sleeve gastrectomy (LSG) modifies the anatomy of the GI system, modulating nutrient transit and impacting gut physiology. The procedure is known to produce a long-term reduction in body weight and a decrease in the blood glucose level, both of which are relevant to obesity-related type 2 diabetes mellitus and cardiovascular disease. Some studies have pointed to the beneficial effect of surgery on the microbiotic diversity as a partial contribution to the resolution of the metabolic status of these patients [9] and subsequent improvement their QOL.

In this issue, Kazzi and her colleagues [10] focus on the possible effect of exogenous supplemental diet with probiotics and prebiotics after LSG on the QOL. This report suggests that surgical manipulation of the stomach during LSG may induce challenges within the bowel and investigated whether these may be alleviated by the use of probiotics and prebiotics. Although the study confirmed that bariatric surgery produces weight loss and improvement in comorbid conditions associated with obesity and of the QOL in all patients following surgery, it failed to show any significant difference in QOL between patients using prebiotics and those taking placebo for 3 months post operatively. Bariatric surgical intervention for weight loss, by itself, impacts the gut microbiota composition. Further trials are needed to draw conclusions about the role of microbial diversity in obesity.

\section{References}

1. Oria HE, Moorehead MK. Bariatric analysis and reporting outcome system (BAROS). Obes Surg 1998;8:487-99.

2. Livingston EH, Fink AS. Quality of life: Cost and future of bariatric surgery. Arch Surg 2003;138:383-8.

3. Van Hout G. Psychosocial effects of bariatric surgery. Acta Chir Belg 2005;105:40-3.

4. Robert M, Denis A, Badol-Van Straaten P, et al. Prospective longitudinal assessment of change in health-related quality of life after adjustable gastric banding. Obes Surg 2013;23:1564-70.

5. Apovian CM, Huskey KW, Chiodi S, et al. Patient factors associated with undergoing laparoscopic adjustable gastric banding vs Roux-en-Y gastric bypass for weight loss. J Am Coll Surg 2013;217:1118-25.

6. Baothman OA, Zamzami MA, Taher I, et al. The role of gut microbiota in the development of obesity and diabetes. Lipids Health Dis 2016;15:108.

7. Sekirov I, Russell SL, Antunes LC, et al. Gut microbiota in 
health and disease. Physiol Rev 2010;90:859-904.

8. Cani PD, Osto M, Geurts L, et al. Involvement of gut microbiota in the development of low-grade inflammation and type 2 diabetes associated with obesity. Gut Microbes 2012; 3:279-88.

9. Seganfredo FB, Blume CA, Moehlecke M, et al. Weight-loss interventions and gut microbiota changes in overweight and obese patients: A systematic review. Obes Rev 2017;18:83251.

10. F Kazzi, N Daher, G Kray et al. Probiotics and prebiotics effect on quality of life after bariatric surgery. Hellenic J Surg 2018;90:121-6. 